\title{
AVALIAÇÃO DE CLASSIFICADORA DE CILINDROS DIVERGENTES PARA TOMATE "CARMEN"
}

\author{
MÁRCIA E. ATARASSI ${ }^{1}$, MARCOS D. FERREIRA ${ }^{2}$, OSCAR A. BRAUNBECK ${ }^{3}$, \\ CELSO L. MORETTI ${ }^{4}$
}

\begin{abstract}
RESUMO: As normas de classificação garantem o mesmo padrão para produtores, atacadistas e consumidores finais, facilitando a comercialização, agregando valor ao produto e diminuindo as perdas. O objetivo deste trabalho foi avaliar uma classificadora de tomates in natura por diâmetro, por meio de cilindros rotativos divergentes com diferentes velocidades de rotação. Foram realizados ensaios para avaliar a eficiência da classificação, conformidade da classificação por diâmetro com a norma do PROGRAMA BRASILEIRO PARA A MODERNIZAÇÃO DA HORTICULTURA (2003) e incidência de danos mecânicos em tomates "Carmen". Os cilindros divergentes apresentaram conformidade de mistura na classificação por diâmetro, nas classes 60 e 80 . Entretanto, para a classe 70, nenhum tratamento esteve em conformidade com a mistura permitida de $10 \%$ de frutos de outras classes. O conjunto de um cilindro liso e outro com helicoide de borracha a $150 \mathrm{rpm}$ obteve a maior eficiência de classificação, com menor perda de massa dos tomates armazenados e atividade respiratória similar aos frutos-controle.
\end{abstract}

PALAVRAS-CHAVE: pós-colheita, classificação, Lycopersicon esculentum Mill, qualidade, atividade respiratória.

\section{PERFORMANCE EVALUATION OF DIVERGENT SIZING CYLINDERS EQUIPMENT FOR “CARMEN” TOMATO}

\begin{abstract}
Grading standards guarantee consistent quality for growers, wholesalers and consumers, helping the trading, adding value to products and reducing postharvest losses. The main purpose of this study was to evaluate a grading diameter system for fresh market tomatoes in a rotative cylinder equipment in different rotation velocities. Sorting efficiency, based on the standard diameter of THE BRAZILIAN PROGRAM FOR THE HORTICULTURE MODERNIZATION (2003) and mechanical damage incidence for "Carmen" tomatoes were evaluated. The divergent cylinders were in accordance with grade mixing in classes 60 and 80 grading. However, for grade 70 no treatment was in accordance with the mixture percentage allowed, which is $10 \%$ of fruit from other classes. The combination of a flat and a helical rubber cylinder, both at $150 \mathrm{rpm}$, had the highest sorting efficiency, showing low weight loss and respiratory activity rates, values similar to the ones found for control treatments.
\end{abstract}

KEYWORDS: post harvest, sizing, Lycopersicon esculentum Mill, quality, respiratory activity.

\section{INTRODUÇÃO}

O Brasil ocupou o nono lugar na produção mundial de tomates em 2007, com 3,36 milhões de toneladas, cultivadas em área de 56,68 mil hectares (FAO, 2008). O Estado de Goiás foi o maior produtor em 2007, com 802.128 toneladas, seguido por São Paulo, com 713.483 toneladas (IBGE, 2008). Apesar desta grande produção, a cadeia produtiva do tomate de mesa apresenta, em diferentes graus, problemas de padronização de classificação. A norma de classificação de produtos

\footnotetext{
${ }^{1}$ Eng ${ }^{\text {a }}$ Agrícola, M.Sc. em Tecnologia Pós-Colheita, Assistente Agropecuária I, CATI - Coordenadoria de Assistência Técnica Integral, Av. Brasil, 2340, Campinas - SP, marcia.atarassi@ cati.sp.gov.br

${ }^{2}$ Pesquisador A, Embrapa Instrumentação Agropecuária, Caixa Postal 741, São Carlos - SP, Fone: (0XX16) 2107.2804, Pesquisador Colaborador, Faculdade de Engenharia Agrícola, UNICAMP, Campinas - SP, marcosferreira@ cnpdia.embrapa.br

${ }^{3}$ Eng ${ }^{\mathrm{o}}$ Industrial, Prof. Doutor, FEAGRI/UNICAMP, Campinas - SP, obraunbeck@ bioetanol.org.br

${ }^{4}$ Eng ${ }^{\mathrm{O}}$ Agrônomo, Doutor, Pesquisador A, Laboratório de Pós-Colheita, Embrapa Hortaliças, Brasília - DF.

Recebido pelo Conselho Editorial em: 6-1-2009

Aprovado pelo Conselho Editorial em: 12-12-2009
} 
agrícolas permite que exista uma linguagem única e transparente para toda a cadeia produtiva. Além disso, FARINA (2003) ressalta que a classificação oferece vantagens, tanto para compradores como para vendedores, pois viabiliza a realização de negócios, independentemente da presença física da mercadoria. Assim, obtêm-se transparência na comercialização, melhores preços para produtores e consumidores, menores perdas e melhor qualidade.

Atualmente, muitos produtores realizam classificação manual, em geral de baixo custo e baixa padronização. Os equipamentos mecânicos de classificação demonstram baixa qualidade no produto beneficiado, como FERRARI (2005) verificou na avaliação de equipamento mecânico de lonas perfuradas. Esse autor constatou que a mistura não apresentou conformidade com a norma em nenhuma classe de tamanho, durante duas safras de colheita. Segundo PELEG (1985), SARGENT et al.(1989a) e SARGENT et al. (1989b), as operações de seleção e de classificação ou a passagem do produto por equipamentos inadequados podem ser os pontos principais na incidência de injúrias mecânicas.

BASSETTO (2006) avaliou a colheita e a venda de pêssegos em 2003 e verificou que a classificação era a etapa mais crítica para danos físicos, apresentando $38 \%$ de frutos com injúrias mecânicas. Nos dois anos seguintes, a incidência de injúrias mecânicas reduziu-se consideravelmente para o nível de $4 \%$ na etapa de classificação, provavelmente devido à conscientização do produtor, após os primeiros resultados de avaliação, da importância do manejo pós-colheita dos frutos.

MAGALHÃES \& FERREIRA (2006) constataram que tomates que passaram por um protótipo de linha de beneficiamento em laboratório e sofreram queda de $30 \mathrm{~cm}$ sobre superfície de caixa plástica, tiveram $3,46 \%$ de perda de massa após 8 dias em armazenamento a $23{ }^{\circ} \mathrm{C}$. Com a redução da altura de queda para $10 \mathrm{~cm}$, a perda de massa foi de $2,63 \%$, indicando que maiores alturas de quedas causam maiores injúrias internas. Além disso, MORETTI \& SARGENT (2000) constataram que a desordem fisiológica causada por impactos alterou o sabor e o aroma de tomates, reduzindo de maneira potencial a aceitação desse produto.

Diante do exposto, o objetivo do presente trabalho foi ajustar e avaliar uma classificadora de tomates de mesa, que utiliza cilindros divergentes com distintas velocidades de rotação, como alternativa aos sistemas comerciais, avaliando-se seu desempenho e sua influência na qualidade do fruto.

\section{MATERIAL E MÉTODOS}

\section{Equipamento de classificação}

O equipamento de classificação $(0,65 \mathrm{~m}$ de largura, $1,85 \mathrm{~m}$ de comprimento e $0,90 \mathrm{~m}$ de altura) é formado por perfis metálicos e por um par de cilindros de $1,50 \mathrm{~m}$ de comprimento e 0,114 m de diâmetro, montados em mancais de rolamentos de esferas de forma divergente (ângulo de $1,8^{\circ}$ ) e girando no mesmo sentido. A velocidade e o sentido de rotação dos cilindros foram controlados por um motor elétrico (WEG, modelo $189 \mathrm{~W}$ ), ligado a um inversor de frequência (WEG, CFW 08). Acima dos cilindros, foi afixada uma calha para evitar que os tomates saíssem por cima do sistema. Abaixo dos cilindros, foram posicionadas divisórias de papelão ondulado espaçadas em $0,10 \mathrm{~m}$ entre si, formando 12 saídas de classificação, identificadas sequencialmente por letras de A a M (Figuras 1a e 1b). Os frutos submetidos à classificação caíam entre as aberturas dos dois cilindros e eram depositados em recipientes plásticos protegidos com espuma. As divisórias foram necessárias, pois à medida que se aumentava a velocidade de rotação, a classificação ocorria em aberturas maiores. 

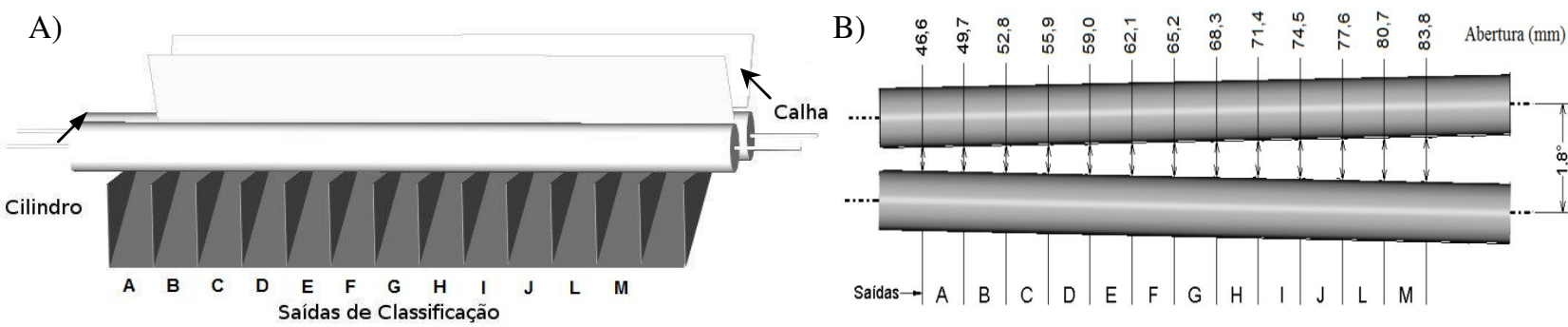

FIGURA 1. Equipamento de classificação com cilindros divergentes. a) Indicação dos principais componentes do equipamento: cilindros, calha e saídas de classificação. b) Vista superior com os valores de abertura $(\mathrm{mm})$ entre as saídas de classificação e o ângulo entre os cilindros. Grading equipment with divergent cylinders. a) Main components of equipment: cylinders, classification channel and outputs. b) Top view of the equipment showing dimensions $(\mathrm{mm})$ between outputs of classification and cylinders angle.

Os tipos de cilindros empregados foram: cilindro com cerdas, composto de um cilindro central de Polietileno de Alta Densidade (PAD), onde são inseridas as cerdas retas de Tereftalato de Polietileno (PET) de fios de $0,15 \mathrm{~mm}$ de diâmetro e $25 \mathrm{~mm}$ de comprimento; cilindro liso de tubo de Cloreto de Polivinila (PVC), e cilindro com helicoide, composto de tubo de PVC com uma helicoide de borracha fixada (altura de $0,01 \mathrm{~m}$ e passo de $0,10 \mathrm{~m}$ ).

Os 15 tratamentos avaliados foram formados pela combinação de cinco velocidades de rotação: $50 \mathrm{rpm}\left(5,24 \mathrm{rad} \mathrm{s}^{-1}\right) ; 100 \mathrm{rpm}\left(10,47 \mathrm{rad} \mathrm{s}^{-1}\right) ; 150 \mathrm{rpm}\left(15,71 \mathrm{rad} \mathrm{s}^{-1}\right) ; 200 \mathrm{rpm}$ $\left(20,94 \mathrm{rad} \mathrm{s}^{-1}\right)$, e $250 \mathrm{rpm}\left(26,18 \mathrm{rad} \mathrm{s}^{-1}\right)$, e três conjuntos de cilindros: dois cilindros com cerdas CC (Figura 2a), um cilindro com cerdas e um liso - CL (Figura 2b); e um cilindro liso e outro com helicoide - LH (Figura 2c).
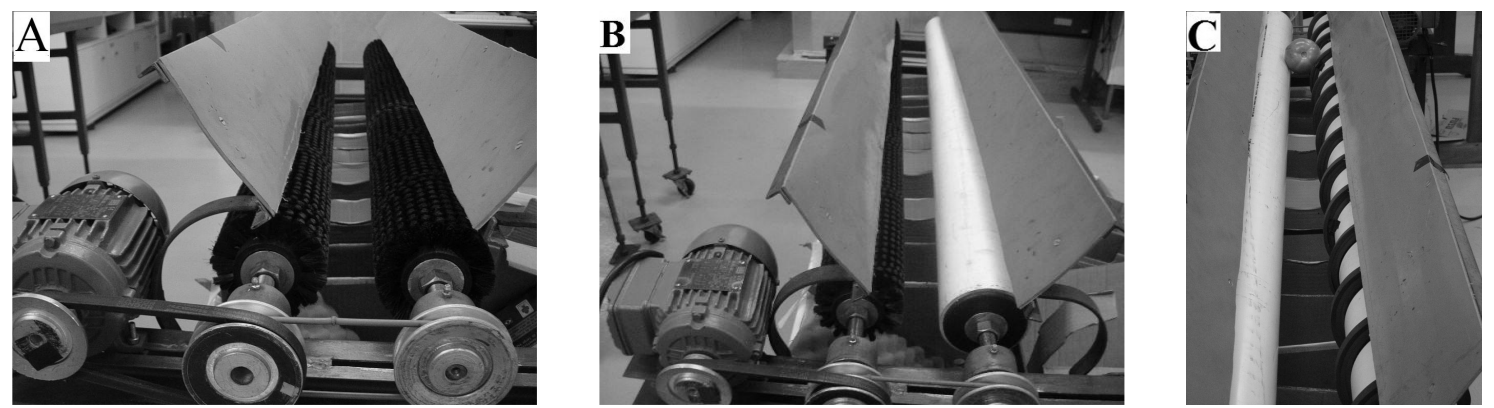

FIGURA 2. Equipamento de classificação e os conjuntos de cilindros avaliados. a) Dois cilindros com cerdas - CC; b) Um cilindro com cerdas e um liso - CL; c) Um cilindro liso e outro com helicoide - LH. Grading equipment showing the evaluated cylinders sets. a) Two cylinders with bristles - CC b) Bristle and flat cylinders - CL c) Flat and helical rubber cylinders - $\mathbf{L H}$.

\section{Definição de saídas e avaliação de classificação}

Para definir as saídas de classificação e avaliar a classificação de cada tratamento, aplicou-se a mesma metodologia utilizada por JARIMOPAS et al. (2007), empregando-se tomates (Lycopersicon esculentum Mill.) da cultivar 'Carmen', escolhendo-se 30 frutos de três classes de tamanhos diferentes (classe 60: diâmetro-padrão de 60 a 70 mm; classe 70: diâmetro-padrão de 70 a $80 \mathrm{~mm}$, e classe 80: diâmetro-padrão de 70 a $80 \mathrm{~mm}$ ), seguindo a norma de classificação do PROGRAMA BRASILEIRO PARA A MODERNIZAÇÃO DA HORTICULTURA (2003). 
Esses 90 tomates foram numerados, pesados e medidos em três sentidos diferentes (duas medições equatoriais de maior e menor diâmetro e uma longitudinal) por meio de paquímetro digital Mitutoyo 500-144B. Os frutos foram misturados e colocados individual e manualmente de modo contínuo pelo lado de menor abertura entre cilindros, e com um cronômetro digital, mediu-se o tempo total para classificar os 90 tomates. Em seguida, foi anotado o número do tomate classificado em cada saída, realizando-se cinco repetições. Por meio desse ensaio, foram relacionadas as saídas de classificação com as classes 60; 70 e 80, de acordo com as maiores frações de pureza de cada classe. A fração de pureza $\left(\mathrm{P}_{\mathrm{gi}}\right)$ é definida pela razão entre a quantidade de frutos da classe i, classificados na saída, e o total da saída, que representa o índice de frutos de cada classe na saída.

Após a definição das saídas de cada tratamento, avaliou-se a classificação realizada pelo sistema, verificando-se a conformidade com a norma de classificação do tomate, do PROGRAMA BRASILEIRO PARA A MODERNIZAÇÃO DA HORTICULTURA (2003). Assim, aplicou-se a mesma metodologia descrita por FERREIRA et al. (2005), comparando-se os limites-padrão com os limites superior e inferior de diâmetro dos tomates classificados, calculados por meio do teste t, com intervalo de confiança de $90 \%$. Foi adotado esse valor de confiabilidade, pois, segundo a norma do PROGRAMA BRASILEIRO PARA A MODERNIZAÇÃO DA HORTICULTURA (2003), tolera-se uma mistura com até $10 \%$ de tomates de outras classes, desde que pertençam à classe imediatamente superior e/ou inferior da classe declarada no rótulo da embalagem (PROGRAMA BRASILEIRO PARA A MODERNIZAÇÃO DA HORTICULTURA, 2003).

Também aplicou-se o teste t (nível de confiança de 95\%) para verificar se a mistura era de até 10\%. Ainda outro parâmetro analisado foi a eficiência de classificação $\left(\mathrm{E}_{\mathrm{w}}\right)$, definida por PELEG (1985), que indica a qualidade da classificação em relação à taxa de entrada e saída dos frutos [eq.(1)]:

$$
E_{w}=\frac{P_{g 60} G_{60}+P_{g 70} G_{70}+P_{g 80} G_{80}}{Q}
$$

em que,

$\mathrm{E}_{\mathrm{w}}$ - eficiência de classificação, \%;

$\mathrm{P}_{\mathrm{g} 60}, \mathrm{P}_{\mathrm{g} 70}$ e $\mathrm{P}_{\mathrm{g} 80}$ - frações de pureza das classes 60; 70 e 80, respectivamente. Fração de pureza $\left(\mathrm{P}_{\mathrm{gi}}\right)$ é a razão entre a quantidade de frutos da classe $\mathrm{i}$, classificados na saída, e o total da saída;

$\mathrm{Q}$ - taxa de entrada de tomates na classificadora, $\mathrm{kg} \mathrm{h}^{-1}$, e

$\mathrm{G}_{60}, \mathrm{G}_{70}$ e $\mathrm{G}_{80}$ - taxa de saída, nas classes 60; 70 e 80, respectivamente, $\mathrm{kg} \mathrm{h}^{-1}$.

\section{Perda de massa e atividade respiratória após o processo de classificação}

Para avaliar a qualidade dos tomates submetidos à classificadora, mediu-se a perda de massa e a atividade respiratória em frutos-controle e em tomates que passaram pelo equipamento, utilizando os três conjuntos de cilindros, nas duas velocidades de rotação de maior eficiência de classificação: CC a 50 e 100 rpm; CL a 50 e 250 rpm, e LH a 150 e 200 rpm.

A produção de $\mathrm{CO}_{2}$ foi determinada colocando-se cinco tomates por tratamento em um recipiente com capacidade de 3,5 $\mathrm{L}$, hermeticamente fechado, sob as condições de ambiente de $24,76 \pm 3,98^{\circ} \mathrm{C}$ e umidade relativa de $50,44 \pm 13,87 \%$.

Coletou-se $1 \mathrm{~mL}$ da atmosfera gasosa do frasco, com a ajuda de uma seringa hipodérmica, por meio de um septo de silicone adaptado às tampas dos frascos, injetou-se a amostra em cromatógrafo da marca VARIAN, modelo CG 3400, e os resultados foram expressos em $\mathrm{mg} \mathrm{CO}_{2} \mathrm{~kg}^{-1} \mathrm{~h}^{-1}$. A perda de massa foi monitorada durante 14 dias de armazenamento, em 30 frutos por tratamento. A perda de massa $(\%)$ foi expressa utilizando a relação entre a diferença entre massa inicial e final. $\mathrm{Na}$ comparação dos tratamentos, utilizou-se o teste de Tukey (nível de probabilidade de 5\%). 


\section{RESULTADOS E DISCUSSÃO}

\section{Definição das saídas de classificação}

Segundo os dados do ensaio de classificação, designaram-se as saídas de cada classe de tamanho, de acordo com o conjunto de cilindros e com a velocidade de rotação (Tabela 1). Notou-se que, nas velocidades de rotação maiores, os frutos caíam em saídas com aberturas maiores. Esse comportamento ocorre pelo movimento balístico do produto, causada pela maior força longitudinal sobre o tomate quando se aumenta a velocidade de rotação.

No caso do conjunto de cilindros CC e CL, outro fator também influenciava na classificação: uma pequena flexão das cerdas causava a classificação em saídas de aberturas menores, em comparação ao conjunto de cilindros LH, que eram rígidos.

TABELA 1. Saídas adotadas para cada classe de tamanho e conjunto de cilindros, nas velocidades de rotação de 50; 100; 150; 200 e 250 rpm, após o ensaio de classificação dos tomates das classes 60; 70 e 80. Outputs taken for each class size and cylinders, in rotation velocities of 50, 100, 150, 200 and $250 \mathrm{rpm}$, after classification trials, on classes 60, 70 and 80 .

\begin{tabular}{ccccc}
\hline \multirow{2}{*}{ Velocidade de Rotação (rpm) } & \multirow{2}{*}{ Classe } & \multicolumn{3}{c}{ Conjunto de Cilindros* } \\
\cline { 3 - 5 } & & CC & CL & LH \\
\hline \multirow{2}{*}{50} & 60 & A-B & A-B & B-D \\
& 70 & C & C-D & E-G \\
& 80 & D-G & E-G & H-L \\
\hline \multirow{2}{*}{100} & 60 & A-B & A & B-D \\
& 70 & C & B-C & E-G \\
& 80 & D-G & D-G & H-L \\
\hline \multirow{2}{*}{150} & 60 & A-B & A-B & C-E \\
& 70 & C-D & C-D & F-H \\
& 80 & E-H & E-G & I-L \\
\hline \multirow{2}{*}{200} & 60 & A-B & A-C & C-E \\
& 70 & C-D & D-E & F-H \\
& 80 & E-H & F-H & I-M \\
\hline \multirow{2}{*}{250} & 60 & A-B & A-D & D-F \\
& 70 & C-E & E-F & G-H \\
& 80 & F-I & G-J & I-M \\
\hline
\end{tabular}

*CC - dois cilindros com cerdas; CL - um cilindro com cerdas e um liso; LH - um cilindro liso e outro com helicoide.

\section{Avaliação de classificação}

Verificou-se que, para as classes 60 e 70, o intervalo das amostras estava contido no intervalo-padrão em todas as velocidades de rotação de todos os conjuntos, atendendo à norma em relação ao tamanho, segundo metodologia de FERREIRA et al. (2005) (Tabela 2).

Na classe 80, a classificação de tamanho esteve de acordo com a norma nos tratamentos CC a 50; 150 e $250 \mathrm{rpm}$; CL a 50 e $250 \mathrm{rpm}$, e em todas as velocidades do tratamento LH. Na classe 60, notou-se que, no tratamento $\mathrm{CC}$, os limites inferior e superior de diâmetro diminuíram com o aumento da velocidade de rotação de 50 para $200 \mathrm{rpm}$, enquanto, no tratamento CL, esses valores tiveram acréscimo com o aumento da velocidade de rotação de 50 para $150 \mathrm{rpm}$, e depois apresentaram decréscimo nas velocidades de 200 e $250 \mathrm{rpm}$. Para o tratamento CC, as classes 70 e 80 apresentaram intervalo variável em relação à velocidade de rotação dos cilindros com cerdas, e no tratamento CL, os limites dos intervalos aumentaram quando se variou a velocidade de rotação de 150 para $250 \mathrm{rpm}$. No tratamento $\mathrm{LH}$, o aumento da velocidade de rotação de 50 para 100 resultou em redução dos limites de diâmetro de todas as classes. A variação de 100 para $150 \mathrm{rpm}$ e de 200 para 250 rpm causou aumento de todos os intervalos. 
TABELA 2. Limites inferior e superior médios de diâmetro das classes $60 ; 70$ e 80 calculado pelo teste $\mathrm{t}(\mathrm{p}<0,10)$, obtidos pelo ensaio de classificação com dois cilindros com cerdas (CC), um cilindro com cerdas e um cilindro liso (CL), e um cilindro liso e um cilindro com helicoide (LH) a 50;100;150; 200 e $250 \mathrm{rpm}$. Lower and upper average diameter limits of classes 60,70 and 80 calculated by $t$ test $(p<0.10)$, obtained by classification trial on two bristle cylinders (CC), a cylinder with bristles and a flat cylinder (CL) and a flat cylinder and a helical rubber one (LH), at 50; 100; 150; 200 and 250 rpm.

\begin{tabular}{ccccc}
\hline \multirow{2}{*}{$\begin{array}{c}\text { Conjunto de } \\
\text { Cilindros }\end{array}$} & $\begin{array}{c}\text { Velocidade de Rotação } \\
(\mathrm{rpm})\end{array}$ & 60 & 70 & \multicolumn{3}{c}{ Classes } \\
\cline { 2 - 5 } & 50 & $66,66-69,56$ & $71,50-75,28$ & $80,29-82,76$ \\
$\mathrm{CC}$ & 100 & $65,78-68,65$ & $71,02-74,40$ & $79,13-81,94^{*}$ \\
& 150 & $65,50-68,45$ & $72,40-75,53$ & $80,78-83,46$ \\
& 200 & $64,30-67,28$ & $71,52-74,53$ & $79,56-82,57^{*}$ \\
& 250 & $64,52-68,28$ & $71,95-74,82$ & $80,85-83,79$ \\
\hline \multirow{4}{*}{ CL } & 50 & $63,52-65,62$ & $76,73-79,19$ & $80,63-82,44$ \\
& 100 & $65,01-67,98$ & $71,76-75,15$ & $79,81-82,69^{*}$ \\
& 150 & $66,40-69,82$ & $72,35-77,09$ & $77,88-81,04^{*}$ \\
& 200 & $65,28-68,63$ & $73,70-76,96$ & $79,14-82,59^{*}$ \\
& 250 & $64,80-67,56$ & $75,09-77,70$ & $81,53-84,00$ \\
\hline \multirow{4}{*}{ LH } & 50 & $64,24-67,14$ & $72,79-75,53$ & $81,18-83,83$ \\
& 100 & $63,31-66,27$ & $71,64-74,45$ & $80,00-82,79$ \\
& 150 & $64,18-66,34$ & $72,62-75,12$ & $81,33-83,31$ \\
*Limite inferior menor que o padrão estabelecido pela norma brasileira. & & $80,24-82,49$ \\
& 200 & $63,50-65,56$ & $71,14-73,69$ & $80,69-83,19$ \\
\hline
\end{tabular}

Verificou-se que houve aumento de $32,8 \%$ da fração de pureza da classe 60 quando se aumentou a velocidade de rotação de 50 para $200 \mathrm{rpm}$, na classificação com o conjunto de cilindros CC (Figura 3). Para a classe 70, ocorreu decréscimo da fração de pureza quando se aumentou a rotação de 100 para $200 \mathrm{rpm}$, com redução de 19,3\%. Portanto, o aumento da velocidade de rotação fez com que mais frutos de outros tamanhos fossem classificados na saída da classe 70, causando a redução de sua fração de pureza. Na classe 80, a fração de pureza nas velocidades de rotação de 150 e $250 \mathrm{rpm}$ foi $18,4 \%$ maior que a $100 \mathrm{rpm}$. A fração de pureza do tratamento CL $50 \mathrm{rpm}$ foi 38,5\% maior que o valor de CL $150 \mathrm{rpm}$ (Figura 4). Para a classe 70, o tratamento CL 50 rpm obteve valor $54,8 \%$ maior que os tratamentos CL 100 e $200 \mathrm{rpm}$, e em relação ao tratamento CL $150 \mathrm{rpm}$, a diferença foi de $94,6 \%$. Na classe 80 , o tratamento CL $250 \mathrm{rpm}$ apresentou a maior fração de pureza, sendo $51 \%$ maior que o tratamento CL $150 \mathrm{rpm}$. Notou-se que a fração de pureza da classe 60 aumentou quando houve acréscimo da velocidade de rotação de 50 para $200 \mathrm{rpm}$ no conjunto de cilindros LH (Figura 5). Para a classe 70, também houve aumento da fração de pureza quando se variou a velocidade de 100 para $150 \mathrm{rpm}$, observando-se que os tratamentos LH 150 e $200 \mathrm{rpm}$ foram $38 \%$ maiores que a $100 \mathrm{rpm}$.

SARGENT et al. (1991) observaram valores de mistura acima de 10\% para a menor classe analisada (diâmetro de 57 a $65 \mathrm{~mm}$ ) em apenas uma das variedades avaliadas na classificação por correias perfuradas. As outras classes estavam em acordo com os padrões de classificação vigentes no Estado da Flórida, Estados Unidos. No Brasil, FERRARI (2005) avaliou um sistema de lonas perfuradas que utiliza o mesmo princípio das correias perfuradas, que classificam o tomate por meio de um orifício de diâmetro preestabelecido. Esse autor verificou que o equipamento não realizava a classificação de acordo com as normas brasileiras.

No sistema de cilindros divergentes, a classificação com o conjunto $\mathrm{CC}$ apresentou mistura estatisticamente menor que 0,10 , na classe 60, no tratamento CC $200 \mathrm{rpm}$, e na classe 80, nos 
tratamentos CC 50; 150 e 250 rpm (Figura 3). Na classificação com o conjunto CL, a classe 60 a 50 e $100 \mathrm{rpm}$, e a classe 80 a 100 e $250 \mathrm{rpm}$ também estiveram em acordo com a mistura permitida (Figura 4). O mesmo ocorreu no conjunto LH, na classificação da classe 60, nas velocidades de rotação de 100; 150 e $200 \mathrm{rpm}$, e na classe 80, a 50; 100; 150 e $250 \mathrm{rpm}$ (Figura 5). Nos três conjuntos de cilindros (CC, CL e LH) e em todas as velocidades, a classificação da classe 70 não esteve em acordo com a norma.

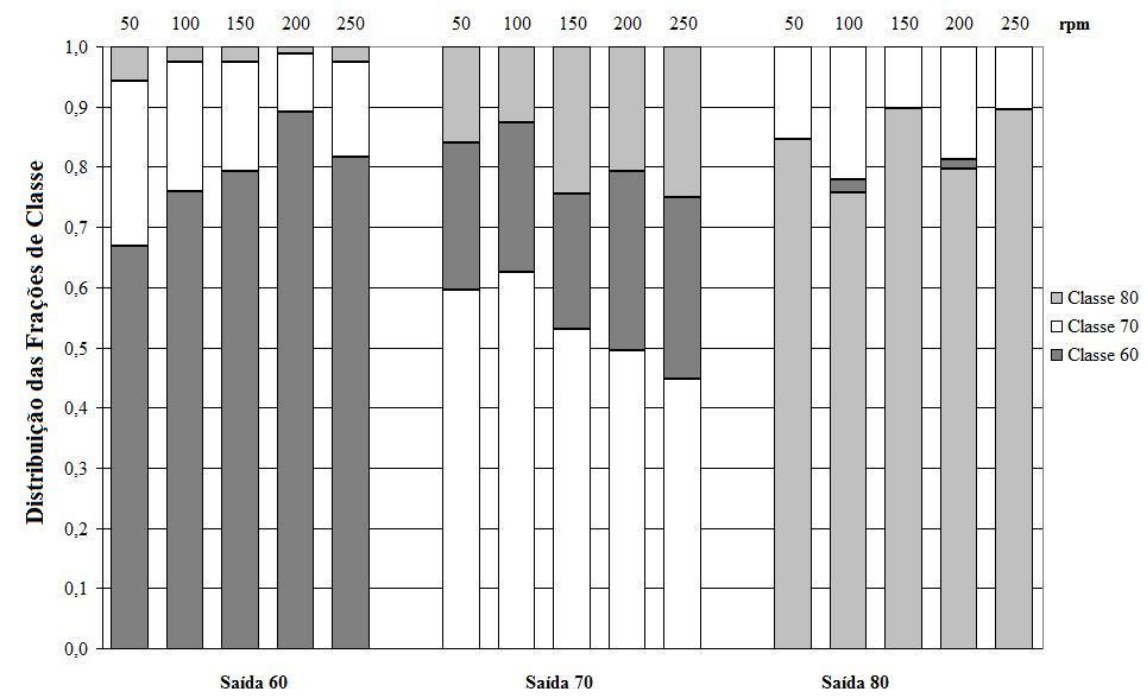

FIGURA 3. Distribuição de frações de classes em cada saída, obtido pelo ensaio de classificação com dois cilindros com cerdas (CC), a 50; 100; 150; 200 e $250 \mathrm{rpm}$. A fração de classe foi calculada pela razão entre a quantidade de frutos da classe $60 ; 70$ e 80, classificados na saída, e o total da saída. Fraction classes distribution in each output, obtained by classification trials on two bristle cylinders $(\mathrm{CC}), 50,100$, 150,200 and $250 \mathrm{rpm}$. The class fraction was calculated by the ratio between the amount of fruit in class 60,70 and 80 placed in output and total output.

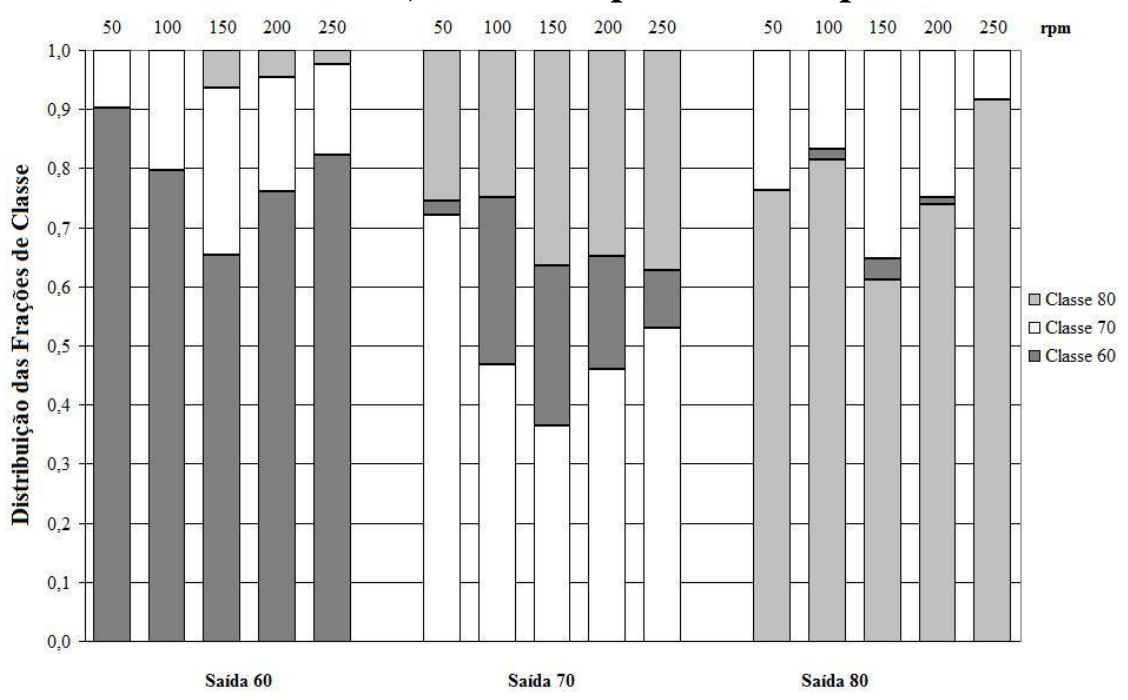

FIGURA 4. Distribuição de frações de classes em cada saída, obtida pelo ensaio de classificação com um cilindro com cerdas e um cilindro liso (CL), a 50; 100; 150; 200 e $250 \mathrm{rpm}$. A fração de classe foi calculada pela razão entre a quantidade de frutos da classe 60; 70 e 80, classificados na saída, e o total da saída. Fraction classes distribution in each output, obtained by classification trials with a bristle cylinder and a flat cylinder (CL) 50, 100, 150, 200 and $250 \mathrm{rpm}$. The class fraction was calculated by the ratio between the amount of fruit in class 60,70 and 80 placed in output and total output. 


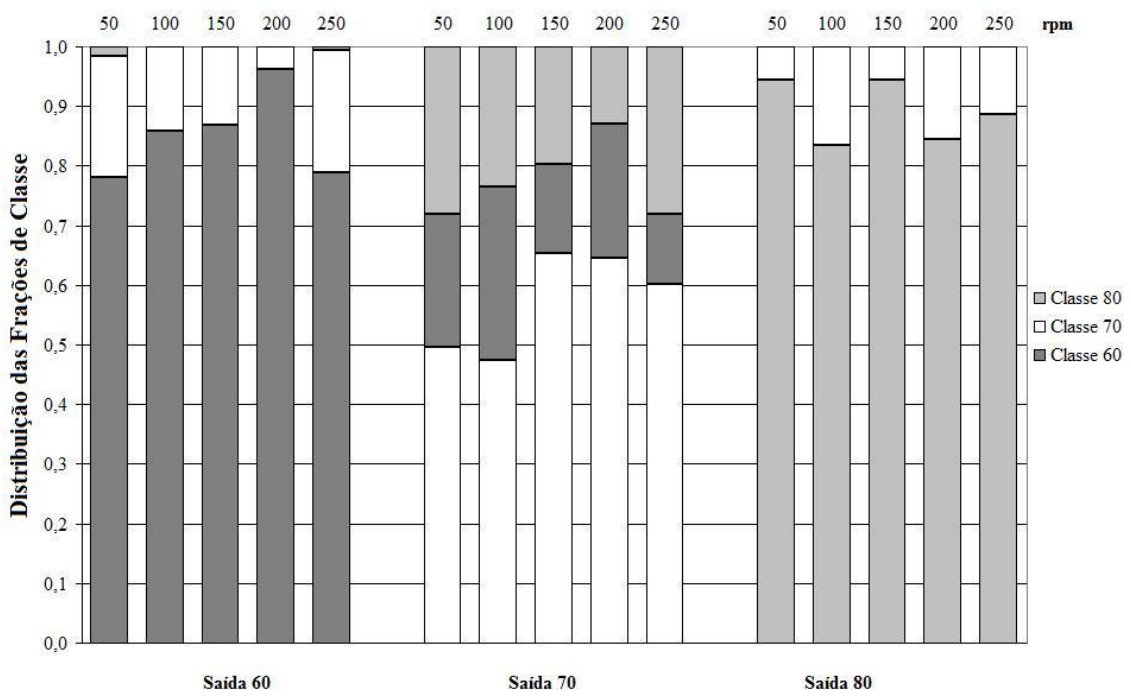

FIGURA 5. Distribuição de frações de classes em cada saída, obtido pelo ensaio de classificação com um cilindro liso e um cilindro com helicoide (LH), a 50; 100; 150; 200 e $250 \mathrm{rpm}$. A fração de classe foi calculada pela razão entre a quantidade de frutos da classe 60; 70 e 80, classificados na saída, e o total da saída. Fraction class distribution in each output, obtained by classification trials with a flat and a helical rubber cylinder (LH) 50, 100, 150, 200 and $250 \mathrm{rpm}$. The class fraction was calculated by the ratio between the amount of fruit in class 60,70 and 80 placed in output and total output.

Verificou-se que a eficiência de classificação foi maior para o tratamento $\mathrm{CC}$, a $50 ; 100$ e $150 \mathrm{rpm}$, e que houve redução a velocidades superiores a essas (Figura 6). Assim, a eficiência a $100 \mathrm{rpm}$ foi $18 \%$ maior que a $250 \mathrm{rpm}$. A classificação por meio do tratamento CL obteve a maior eficiência de classificação a $50 \mathrm{rpm}$, apresentando valor aproximado de $78 \%$, sendo $43 \%$ superior a $150 \mathrm{rpm}$. Para o tratamento LH, os melhores resultados foram a 150 e $200 \mathrm{rpm}$, com eficiências de classificação de 80 e $78 \%$, respectivamente. Comparando os tratamentos de cilindros entre si, conclui-se que, a 150; 200 e $250 \mathrm{rpm}$, a eficiência de classificação foi maior quando se utilizou o tratamento LH, observando-se que esse tratamento foi, em média, $19 \%$ e $27 \%$ superior aos tratamentos CC e CL, respectivamente (Figura 6).

Observou-se que, nos tratamentos CC e CL, em todas as velocidades de rotação, a maioria dos frutos girava em torno de um eixo equatorial, que provocava a classificação pelo comprimento de alguns tomates e reduzia a eficiência de classificação (Figura 7). THROOP et al. (2001) concluíram que a posição mais estável de giro para as maçãs será com seu centro de gravidade na posição mais baixa, ou seja, girando em torno de seu eixo de maior dimensão. A mesma situação ocorreu na classificação com os tratamentos CC e CL, pois os tomates 'Carmen' têm formato redondo, e seu maior eixo é o diâmetro equatorial (Figura 8a). Para mudar o eixo de giro, é necessário aplicar uma força no mesmo sentido do eixo de rotação de menor energia, capaz de modificar o momento de giro do produto. No caso do cilindro do tratamento LH, a helicoide aplicava essa força (Figura 8b), provocando o alinhamento perpendicular ao diâmetro dos frutos, resultando nas maiores eficiências de classificação nas velocidades de rotação acima de 150 rpm. 


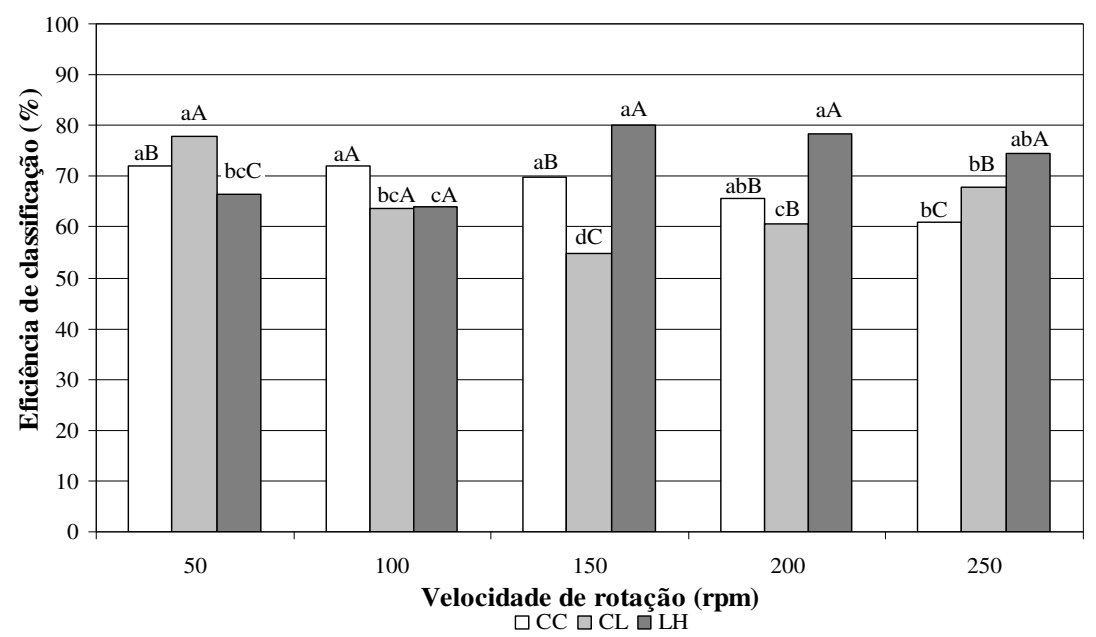

FIGURA 6. Valores médios de eficiência de classificação obtidos pela classificação de tomates submetidos aos tratamentos CC, CL e CH, e nas velocidades de rotação de 50; 100; 150; 200 e $250 \mathrm{rpm}$. Mesma letra minúscula não há diferença entre velocidades de rotações; mesma letra maiúscula não há diferença entre conjunto de cilindros, teste de Tukey, $\mathrm{p}<0,05$. Average values of sorting efficiency obtained by tomatoes classification subjected to treatments $\mathrm{CC}, \mathrm{CL}$ and $\mathrm{CH}$, and rotation velocities of 50, 100, 150, 200 and $250 \mathrm{rpm}$. Same small letter there is no difference between speeds of rotation; same capital letter there is no difference between number of cylinders, Tukey test, $p<0.05$.

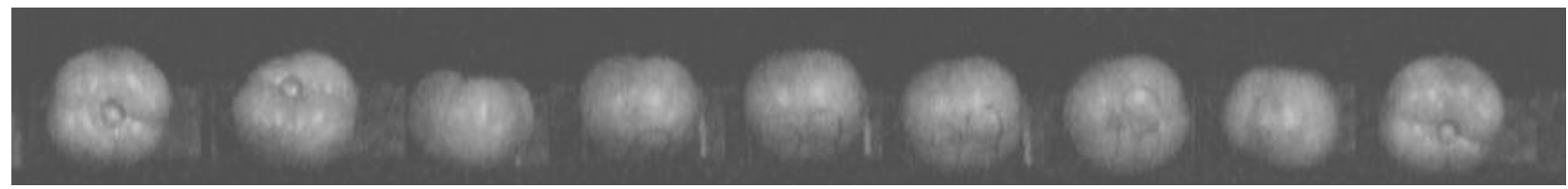

FIGURA 7. Exemplo de movimentação de um tomate sobre os dois cilindros com cerdas (CC) em diferentes instantes. Durante a movimentação longitudinal do fruto sobre os cilindros, ocorre giro de $360^{\circ}$ da inserção do pedúnculo floral em torno do seu eixo equatorial. : Example of how a tomato moves itself on two bristle cylinders (CC) at different moments. During longitudinal fruit movement on the cylinders there is a $360^{\circ}$ rotation of floral insertion around its equatorial axis.

A)

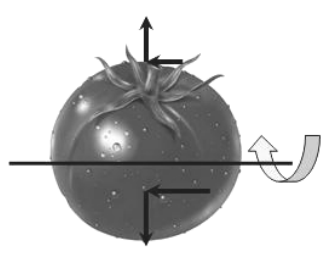

B)

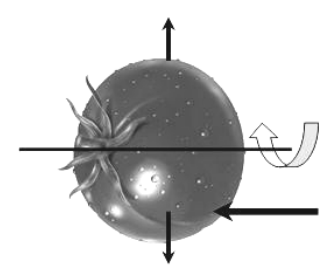

FIGURA 8. Forças atuantes no tomate durante a passagem pelo equipamento. a) Com dois cilindros com cerdas (CC) e um cilindro com cerda e outro liso (CL), existe uma força longitudinal, mas sua magnitude não é suficiente para mudar o eixo de giro do tomate; b) Com um cilindro liso e outro com helicoide ( $\mathrm{LH})$, a força longitudinal atuante é suficiente para mudar o eixo de giro e causa a classificação pelo diâmetro. Acting forces through the equipment. a) On two bristle cylinders (CC) and a bristle and a flat cylinder (CL) there is a longitudinal force, but its magnitude is not enough to change the spin axis of the tomato; b) Flat and a helical rubber cylinder (LH) the acting longitudinal force is enough to change the spin axis and to cause the classification by the diameter. 


\section{Perda de massa e atividade respiratória após o processo de classificação}

Durante o período de armazenamento, observou-se que, a partir do oitavo dia, os frutos do tratamento CL $150 \mathrm{rpm}$ apresentaram as maiores perdas de massa. Os tomates do tratamento LH $150 \mathrm{rpm}$ obtiveram os menores valores, e o restante dos tratamentos obteve valores intermediários entre os dois (Tabela 3). A maior perda de massa do tratamento CL $50 \mathrm{rpm}$ poderia ser justificada devido à abrasão aplicada em áreas de contato diferentes, a qual causa a retirada da película protetora da casca. No caso dos conjuntos CC e CL, a área de contato era maior que no conjunto de rolo liso e com helicoide ( $\mathrm{LH})$, pois as cerdas ficavam em contato em uma área em torno do eixo equatorial.

TABELA 3. Perda de massa (\%) de tomates 'Carmen' durante 14 dias, após a classificação pelo equipamento. Weight loss $(\%)$ of 'Carmen' tomatoes during storage for 14 days after the classification by the equipment with divergent cylinders.

\begin{tabular}{lcllllll}
\hline \multirow{2}{*}{ Tratamentos $^{(1)}$} & \multicolumn{7}{c}{ Tempo de Armazenamento (dias) } \\
\cline { 2 - 8 } & \multicolumn{1}{c}{2} & \multicolumn{1}{c}{4} & 6 & 8 & 10 & 12 & 14 \\
\hline Controle & $1,53 \mathrm{~b}{ }^{(2)}$ & $2,64 \mathrm{ab}$ & $3,52 \mathrm{ab}$ & $4,38 \mathrm{ab}$ & $5,16 \mathrm{ab}$ & $5,96 \mathrm{ab}$ & $6,69 \mathrm{ab}$ \\
CC 50 rpm & $1,44 \mathrm{~b}$ & $2,55 \mathrm{~b}$ & $3,39 \mathrm{~b}$ & $4,39 \mathrm{ab}$ & $5,15 \mathrm{ab}$ & $5,99 \mathrm{ab}$ & $6,81 \mathrm{ab}$ \\
CC 100 rpm & $1,35 \mathrm{~b}$ & $2,38 \mathrm{~b}$ & $3,35 \mathrm{~b}$ & $4,42 \mathrm{ab}$ & $5,31 \mathrm{ab}$ & $6,08 \mathrm{ab}$ & $6,83 \mathrm{ab}$ \\
CL 50 rpm & $1,83 \mathrm{a}$ & $3,00 \mathrm{a}$ & $3,97 \mathrm{a}$ & $4,81 \mathrm{a}$ & $5,63 \mathrm{a}$ & $6,47 \mathrm{a}$ & $7,25 \mathrm{a}$ \\
CL 250 rpm & $1,63 \mathrm{ab}$ & $2,58 \mathrm{ab}$ & $3,49 \mathrm{ab}$ & $4,27 \mathrm{ab}$ & $5,08 \mathrm{ab}$ & $5,90 \mathrm{ab}$ & $6,66 \mathrm{ab}$ \\
LH 150 rpm & $1,41 \mathrm{~b}$ & $2,46 \mathrm{~b}$ & $3,22 \mathrm{~b}$ & $3,95 \mathrm{~b}$ & $4,65 \mathrm{~b}$ & $5,32 \mathrm{~b}$ & $5,93 \mathrm{~b}$ \\
LH 200 rpm & $1,64 \mathrm{ab}$ & $2,74 \mathrm{ab}$ & $3,54 \mathrm{ab}$ & $4,29 \mathrm{ab}$ & $5,01 \mathrm{ab}$ & $5,72 \mathrm{ab}$ & $6,35 \mathrm{ab}$ \\
\hline
\end{tabular}

${ }^{(1)} \mathrm{CC}$ - dois cilindros com cerdas; CL - um cilindro com cerdas e um liso; LH - um cilindro liso e outro com helicoide. ${ }^{(2)}$ Médias seguidas da mesma letra minúscula na coluna não diferem entre si, pelo teste de Tukey $(\mathrm{p}<5 \%)$.

Depois de duas horas, os tomates classificados pelos tratamentos CL e LH apresentaram valores de atividade respiratória menores que os observados por MOURA et al. (2005) para tomates verde-maduros (53 $\mathrm{mg} \mathrm{CO}_{2} \mathrm{~kg}^{-1} \mathrm{~h}^{-1}$ ) depois de duas horas de colheita. Na quarta hora, foi observada diferença entre a produção de $\mathrm{CO}_{2}\left(\mathrm{ARCO}_{2}\right)$ obtido para os frutos classificados nos tratamentos $\mathrm{CC}$ 50 e $100 \mathrm{rpm}$, que apresentaram os maiores valores (média de $59,21 \mathrm{mg} \mathrm{CO}_{2} \mathrm{~kg}^{-1} \mathrm{~h}^{-1}$ ) em relação ao restante dos tratamentos (Tabela 4). Contudo, após 4 horas de classificação, todos os tratamentos apresentaram atividade respiratória próxima aos valores encontrados por KADER et al. (1978), após 6 horas da colheita, que mediram a $\mathrm{ARCO}_{2}$ entre 55 e $60 \mathrm{mg} \mathrm{kg}^{-1} \mathrm{~h}^{-1}$.

TABELA 4. Atividade respiratória $\left(\mathrm{mg} \mathrm{CO}_{2} \mathrm{~kg}^{-1} \mathrm{~h}^{-1}\right)$ dos tomates 'Carmen' durante 4 horas, após a classificação pelo equipamento com cilindros divergentes. Respiratory activity (mg CO2 kg-1 h-1) of 'Carmen' tomatoes during 4 hours after the classification by the equipment with divergent cylinders.

\begin{tabular}{lllll}
\hline \multirow{2}{*}{ Tratamentos $^{(1)}$} & \multicolumn{4}{c}{ Tempo de Armazenamento (h) } \\
\cline { 2 - 5 } & \multicolumn{1}{c}{1} & \multicolumn{1}{c}{2} & \multicolumn{1}{c}{3} \\
\hline Controle & $39,81 \mathrm{abA}^{(2)}$ & $38,67 \mathrm{abcAB}$ & $35,81 \mathrm{bcAB}$ & $33,52 \mathrm{bB}$ \\
CC 50 rpm & $70,75 \mathrm{aA}$ & $57,77 \mathrm{abA}$ & $56,41 \mathrm{abA}$ & $58,13 \mathrm{aA}$ \\
CC 100 rpm & $67,10 \mathrm{abA}$ & $62,18 \mathrm{aA}$ & $59,76 \mathrm{aA}$ & $60,29 \mathrm{aA}$ \\
CL 50 rpm & $56,18 \mathrm{abA}$ & $35,66 \mathrm{bcAB}$ & $27,29 \mathrm{cB}$ & $34,94 \mathrm{bAB}$ \\
CL 250 rpm & $34,25 \mathrm{bA}$ & $35,63 \mathrm{bcA}$ & $44,07 \mathrm{abcA}$ & $33,71 \mathrm{bA}$ \\
LH 150 rpm & $34,45 \mathrm{bA}$ & $25,90 \mathrm{cA}$ & $24,94 \mathrm{cA}$ & $21,71 \mathrm{bA}$ \\
LH 200 rpm & $42,03 \mathrm{abA}$ & $24,67 \mathrm{cA}$ & $23,13 \mathrm{cA}$ & $26,51 \mathrm{bA}$
\end{tabular}

${ }^{(1)} \mathrm{CC}$ - dois cilindros com cerdas; $\mathrm{CL}$ - um cilindro com cerdas e um liso; LH - um cilindro liso e outro com helicoide. ${ }^{(2)}$ Médias seguidas da mesma letra minúscula na coluna não diferem entre si, pelo teste de Tukey $(\mathrm{p}<5 \%)$. 


\section{CONCLUSÕES}

A classificadora com cilindros apresentou potencial para a classificação de tomates, sendo um sistema simples e de fácil manuseio. Todos os conjuntos de cilindros avaliados atenderam às normas do PROGRAMA BRASILEIRO PARA A MODERNIZAÇÃO DA HORTICULTURA em relação à classificação do diâmetro-padrão. A classe 70 apresentou mistura acima do permitido, que poderia ser corrigida aumentando o comprimento dos cilindros e reduzindo o ângulo de abertura para obter menor variação da abertura ao longo dos cilindros. Em um próximo estudo, para a utilização comercial do equipamento, poder-se-ia adotar o conjunto de cilindro liso e com helicoide (LH) a 150 rpm, que apresentou os melhores resultados de classificação e qualidade pós-colheita de produto.

\section{AGRADECIMENTOS}

À Coordenação de Aperfeiçoamento de Pessoal de Nível Superior (CAPES), à Fundação de Amparo à Pesquisa do Estado de São Paulo (FAPESP), projeto 02/00645-7, ao sistema PRODETAB/EMBRAPA, projeto 55-02/01, e à Faculdade de Engenharia Agrícola - UNICAMP.

\section{REFERÊNCIAS}

BASSETTO, E. Quantificação de danos ao longo da cadeia produtiva de pêssegos e avaliação de métodos alternativos de controle de doenças pós-colheita. 2006. 126 f. Tese (Doutorado em Fitopatologia) - Escola Superior de Agricultura "Luiz de Queiroz”, Universidade de São Paulo, Piracicaba, 2006.

FAO. Faostat Agriculture Data. Disponível em <http://faostat.fao.org>. Acesso em: 18 jul. 2008.

FARINA, E.M.M.Q. Padronização em sistemas agroindustriais. In: ZYLBERSTAJN, D.; SCARE, R.F. Gestão da qualidade no agribusiness. São Paulo: Atlas, 2003. p.18-29.

FERRARI, P.R. Avaliação da qualidade da classificação do tomate de mesa. $2005.147 \mathrm{f}$. Dissertação (Mestrado em Tecnologia Pós-Colheita) - Faculdade de Engenharia Agrícola, Universidade Estadual de Campinas, Campinas, 2005.

FERREIRA, M.D.; KUMAKAWA, M.K.; ANDREUCCETTI, C.; HONÓRIO, S.L.; TAVARES, M.; MATHIAS, M.L. Avaliação de linhas de beneficiamento e padrões de classificação para tomate de mesa na região de Campinas - SP. Horticultura Brasileira, Brasília, v.23, n.4, p.940-944, 2005.

IBGE. INSTITUTO BRASILEIRO DE GEOGRAFIA E ESTATÍSTICA. Banco de dados agregados. Disponível em <http://www.sidra.ibge.gov.br>. Acesso em: 12 jul. 2008.

JARIMOPAS, B.; TOOMSAENGTONG, S.; INPRASIT, C. Design and testing of a mangosteen fruit sizing machine. Journal of Food Engineering, Kidlington, v.79, p.745-751, 2007.

KADER, A.A.; MORRIS, L.L.; STEVENS, M.A.; ALBRIGHT-HOLTON, M. Composition and flavor quality of fresh market tomatoes as influenced by some postharvest handling procedures. Journal of the American Society for Horticultural Science, Alexandria, v.103, n.1, p.6-13, 1978.

MAGALHÃES, A.M.; FERREIRA, M.D. Qualidade dos frutos de tomate de mesa quando submetidos a impacto por ocasião do beneficiamento. Horticultura Brasileira, Brasília, v.24, n.4, p.481-484, 2006.

MORETTI, C.L.; SARGENT, S.A. Alteração de sabor e aroma em tomates causada por impacto. Scientia Agrícola, Piracicaba, v.57, n.3, p.385-388, 2000.

MOURA, M.L.; FINGER, F.L.; MIZOBUTSI, G.P.; GALVÃO, H.L. Fisiologia do amadurecimento na planta do tomate 'Santa Clara' e do mutante 'Firme'. Horticultura Brasileira, Brasília, v.23, n.1, p.81-85, 2005.

PELEG, K. Produce, handling, packing and distribution. Westport: AVI Publishing, 1985. 625 p. 
PROGRAMA BRASILEIRO PARA A MODERNIZAÇÃO DA HORTICULTURA. Normas de classificação do tomate. São Paulo: Centro de Qualidade em Horticultura - CQH/CEAGESP, 2003. (Documentos, 26).

SARGENT, S.A.; BRECHT, J.K.; TALBOT, M.T.; ZOELNER, J.J. Performance of perforated belt sizers as affected by size standards for fresh market tomatoes. Applied Engineering in Agriculture, St. Joseph, v.7, n.6, p.724-728, 1991.

SARGENT, S.A.; BRECHT, J.K.; ZOELLNER, J.J. Assesment of mechanical damage in tomato packing lines. Transactions of the ASAE, St. Joseph, v.30, n.1, p.630-634, 1989a.

SARGENT, S.A.; BRECHT, J.K.; ZOELLNER, J.J.; et al. Reducing mechanical damage tomatoes during handling and shipment. Transactions of the ASAE, St. Joseph, v.30, n.2, p.714-719, 1989b.

THROOP, J.A.; ANESHANSLEY, D.J.; UPCHURCH, B.L.; ANGER, B. Apple orientation on two conveyors: performance and predictability based on fruit shape characteristics. Transactions of the ASAE, St. Joseph, v.44, n.1, p.99-109, 2001. 